\title{
EL DERECHO CONSTITUCIONAL Y LOS NUEVOS MODOS DE ORGANIZACIÓN POLÍTICA ${ }^{1}$
}

\author{
ALBERTO PÉREZ CALVO \\ Catedrático de Derecho Constitucional \\ Universidad Pública de Navarra
}

\author{
SUMARIO \\ I. Una organización social policéntrica \\ II. Cómo abordar el estudio de la nueva si- \\ tuación del Estado \\ III. Conclusiones
}

El texto que sigue es el resultado de una cierta sensación de marginalidad que uno puede sentir cuando se dedica a estudiar aspectos de la integración europea y, sobre todo, cuando se intenta estudiar al Estado, a nuestro Estado, como objeto de las transformaciones inducidas por su pertenencia a la Unión Europea. Creo que estas cuestiones no son marginales sino que, al contrario, afectan directamente a la realidad del Estado en la actualidad. Estas transformaciones del Estado escapan a lo que es clásico en la Teoría del Estado y del Derecho constitucional. Pero cuando se forjaron esos contenidos clásicos, el Estado era otro. Los Estados europeos no habían creado todavía la impresionante construcción de la Unión Europea.

Con las consideraciones que siguen, trato de poner sobre la mesa de discusión la necesidad de abordar de una manera nueva la realidad de un Estado transformado. Es, por tanto, un texto para el debate.

Cuando en estas páginas hablo del Derecho Constitucional, me refiero fundamentalmente a la disciplina del mismo nombre, o sea, a las distintas materias que se incluyen en las directrices de los estudios de Derecho contenidas en el Real Decreto 1424/1990, de 26 de octubre y que se traducen en el conte-

1 Texto completo de la ponencia remitida a las Jornadas sobre Orientación y Método del Derecho Constitucional para su debate en la Comisión sobre aspectos históricos, metajurídicos en general y de Derecho Comparado referentes al Derecho Constitucional. 
nido de los distintos manuales y programas de la asignatura de Derecho constitucional. Evidentemente, la elaboración del Derecho Constitucional como disciplina no se agota en los manuales, como lo demuestra la continua producción científica en monografías, otros libros y revistas. Pero sí que se expresan en los medios antedichos los distintos elementos que constituyen el objeto de esta rama del Derecho recopilados sistemáticamente y tratados de una manera asequible a los estudiantes.

\section{UNA ORGANIZACIÓN SOCIAL POLICÉNTRICA}

El Derecho Constitucional se refiere a la organización del Estado según queda definido por las grandes líneas estructurales definidas en la Constitución. El poder constituyente diseña un Estado de acuerdo con los valores que pretende defender. En ese sentido, desde la primera Constitución en la Historia, la americana de 1779, o desde la primera española de 1812 hasta hoy, las Constituciones han ido diseñando nuevas formas de Estado y elevando a rango jurídico nuevos valores reclamados socialmente al mismo tiempo que se dejaban otros a un lado. Creo que son cuestiones bastante elementales. Sólo quiero insistir en el hecho de que las Constituciones hasta el tercer tercio del siglo pasado respondían a la realidad de unos Estados que eran prácticamente la única forma de organización social por excelencia productora de normas jurídicas aplicables a todos sus ciudadanos. Ciertamente existía el mundo internacional en el que también se producían normas jurídicas de carácter similar, pero su importancia, en general, era mucho menor, a pesar de los esfuerzos realizados con la creación de la Sociedad de Naciones.

Hoy las cosas han cambiado sustancialmente. Los Estados siguen constituyendo las principales manifestaciones de las organizaciones sociales. Y también los principales creadores de Derecho pero, además del Estado hay nuevas organizaciones internacionales creadoras de Derecho. Entre nosotros, la más importante es la Unión Europea, aunque el Consejo de Europa es también muy significativo en materia de la interpretación y defensa de los Derechos fundamentales. Además, la nueva situación internacional, caracterizada por el fenómeno denominado globalización o, mejor, mundialización, muestran nuevos modos de organización política que hemos creado las distintas sociedades.

Se trata de ver la existencia de estos nuevos modos de organización política y la influencia que todo ello puede tener sobre nuestro clásico Derecho Constitucional, aunque voy a insistir, por su importancia, en la Unión Europea.

Como si nos encontráramos en una nueva Edad Media, salvando evidentemente, todas las distancias, podríamos describir la organización social europea y, con menor intensidad, también la de otros continentes, como una poliarquía. En Europa, ciertamente, podemos hablar de una sociedad policéntrica, que se organiza en torno a diversos centros de poder político.

Los españoles, por ejemplo, según lo hemos establecido en nuestra Constitución, como nación y pueblo españoles, tenemos una primera organización 
social en torno a un núcleo de poder, el propio poder soberano del pueblo y los órganos centrales del Estado. Después, en la medida en que hemos ido creando las distintas Comunidades Autónomas, nos hemos ido organizando como nacionalidades, regiones y, en todo caso, como pueblos autónomos, en torno a otros centros constituidos por el pueblo y los órganos decisorios autonómicos. Y no acaban ahí las cosas. El pueblo español, junto con otros pueblos europeos, también se organiza en torno a las Instituciones que tienen en sus manos la capacidad normativa comunitaria dando lugar a una nueva organización política que acoge a todos los europeos nacionales de los Estados miembros de la Unión. Incluso, participamos en otra organización política mundial, la de los ciudadanos de todo el mundo que se manifiesta institucionalmente en la Organización de las Naciones Unidas y en la Comunidad Internacional.

Evidentemente, la intensidad organizativa es muy desigual si hablamos del Estado, de la Unión Europea o de la ONU, de modo que junto a la gran intensidad y extensión de la integración que se produce en el seno del Estado, tenemos la todavía muy débil e inestable integración de la ONU. Si tomáramos como índice de la intensidad de la integración el grado de solidaridad económica que se produce en el seno de las distintas organizaciones, podríamos ver cómo el Estado (al menos el europeo) sigue siendo la organización más integrada y después vienen las demás.

Pero, en definitiva, creo que es muy conveniente que tengamos en cuenta esta organización policéntrica de nuestras sociedades para comprender mejor la complejidad de la organización política en la que nos movemos y cuyos distintos núcleos con capacidad normativa, en función de sus competencias, afectan directamente a nuestra vida individual y social.

\section{CÓMO ABORDAR EL ESTUDIO DE LA NUEVA SITUACIÓN DEL ESTADO}

A partir de lo dicho hasta ahora parece oportuno plantear una primera consideración metodológica que vendría a decir que esta organización compleja (policéntrica) en que nos movemos, debe ser objeto de estudio por parte de los constitucionalistas de modo que constituya un constante telón de fondo en el estudio del Estado y no un objeto de estudio episódico, considerado como una rareza que resulta complicado abordar y que se deja en manos de algunos especialistas.

En muchos casos, esta realidad ha sido olvidada completamente y, en general, hemos estudiado la Unión Europea como algo tangente a los Estados, como si la Unión Europea fuera una entidad existente por sí misma y el Estado se limitara al cumplimiento del Derecho comunitario. Es decir, esta materia ha sido objeto de comentarios marginales al hablar de las fuentes y poco más.

Es muy significativo que el Real Decreto 1424/1990, que he citado antes, "por el que se establece el titulo universitario oficial de Licenciado en Derecho y las directrices generales propias de los planes de estudios conducentes a la 
obtención de aquel», según la presentación que se hace de él, establezca las siguientes directrices respecto del Derecho constitucional:

"La Constitución y el ordenamiento juridico. Organización constitucional del Estado. Derechos y Libertades. El Tribunal Constitucional."

Es cierto que a partir de unas directrices tan amplias como éstas, pueden decirse muchas cosas desde el Derecho Constitucional, pero hay una ausencia, a mi juicio grave, de una mínima consideración del Estado en un marco como el que se está comentando aquí.

Y tan significativo como lo anterior es que el mismo Real Decreto prevea una nueva asignatura, Instituciones de Derecho comunitario, con las siguientes directrices:

"El ordenamiento comunitario. Relaciones con los ordenamientos de los Estados Miembros. Estructura, órganos y poderes de las Comunidades. Garantía judicial de los derechos."

También es significativo que la docencia de esta nueva asignatura se encomiende a las áreas de Derecho Administrativo, Derecho Constitucional y Derecho Internacional Público y Relaciones Internacionales. Esta regla ofrece todas las apariencias de que el criterio fundamental seguido para asignar la docencia no fue una razón estrictamente metodológica.

En cuanto a la segunda directriz de la asignatura Instituciones de Derecho comunitario, referida a las relaciones entre el Ordenamiento comunitario y los internos, es cierto que la directriz pone en contacto la organización comunitaria y la estatal. Sin embargo, creo que este contacto no satisface las necesidades de una comprensión del Estado en el nuevo contexto. Primero porque el impacto de la Unión Europea en los ordenamientos estatales no se limita a las relaciones internormativas, ya que tiene otras muchas manifestaciones. Y segundo, porque creo que, el lugar de estudio de este problema concreto debería estar ubicado en la asignatura en la que se estudian por atribución reglamentaria las fuentes del Derecho.

Creo que la larga y fecunda tradición de las teorías del Estado que se han elaborado tanto en Alemania, como en Francia y en Italia, basadas en el hecho cierto de la realidad dominante del Estado como la forma de organización política fundamental y única fuente normativa relevante hasta la formación de la Sociedad de Naciones y, más tarde, de la Organización de las Naciones Unidas y, en Europa, de la Unión Europea, nos ha condicionado para que desde el Derecho Constitucional sigamos estudiando el Estado, ante todo, como un objeto aislado. Es decir, del mismo modo que si estuviéramos a comienzos del siglo xx. Y todo ello, aún siendo conscientes de que el Estado hoy no es la única forma de organización política fundamental para nuestras vidas puesto que éstas dependen en buena medida de otros nuevos modos de organización política, ni tampoco es la única fuente normativa relevante puesto que junto al Estado tenemos a otras entidades legisladoras como es la Unión europea. Incluso, aun- 
que no con la intensidad de las entidades legisladoras señaladas hasta ahora, la mundialización nos trae otras fuentes de Derecho que también condicionan nuestras sociedades y nuestras vidas.

Esta nueva situación nos fuerza a ir pensando en que habrá que cambiar esta forma de estudio para dar una imagen más cercana de la realidad jurídica de hoy. Efectivamente, el Derecho con el que trabajamos todos los días en nuestro tráfico ordinario procede de diversos ordenamientos pero debe ser aplicado sin necesidad de distinguir la organización política concreta donde se ha creado: la Comunidad Autónoma, el Estado, la Unión Europea o el Derecho internacional. Una revisión de este tipo exigirá probablemente cambios en situaciones académicas que hoy carecen de justificación racional en la organización de nuestros estudios de Derecho y en las directrices reglamentarias de los planes de estudios de Derecho a que me he referido antes.

Nunca antes, los estudiosos del Derecho Constitucional habíamos hecho frente a situaciones como las actuales. Las ideas constitucionales, incluso los textos, circulaban a comienzos del XIX entre los distintos Estados que iban adhiriéndose al constitucionalismo pero la configuración definitiva de la organización estatal, o sea, la aprobación del Derecho que organizaba las relaciones sociales dependía únicamente de cada uno de los Estados soberanos. Hoy el Derecho organizador de nuestra poliédrica vida social ya no procede de un único poder, el del Estado, sino de múltiples centros de producción jurídica, del Estado y de fuera del Estado.

\section{II.1. El ESTADO COMUNITARIO}

Nuestros actuales Estados europeos, que crearon las Comunidades Europeas como instrumento para hacer frente a problemas frente a los que su acción aislada hubiera sido ineficaz, no sólo han dirigido y mantenido la estructura comunitaria y moldeado sus caracteres sino que también han experimentado una profunda influencia y transformación en tanto que miembros de la propia Unión Europea.

Realmente, la organización de varios Estados europeos en las Comunidades de los años 1950 supuso ya un cambio importante para los Estados fundadores. Se esperaba sacar un mejor rendimiento a sus recursos y medios y, como todo el mundo sabe, lo consiguieron plenamente. El Estado clásico, mediante el instrumento de las Comunidades Europeas, lograba solucionar graves problemas históricos y ponía las bases para adaptarse a nuevas circunstancias, incluso, como hoy se puede comprobar, a la situación planteada por los retos de la mundialización. En una palabra, el Estado era más funcional, es decir, desarrollaba mejor los fines que los ciudadanos le habían encomendado en sus respectivas Constituciones.

Pero, al mismo tiempo, el carácter de Estado miembro de esta nueva organización iba a aportarles cambios importantes tanto desde el punto de vista estructural como funcional. Iban a convertirse en Estados comunitarios, una 
nueva manera de ser de esta forma de organización política soberana nacida a caballo entre los siglos XV y XVI. Primero se comprobó la alteración del equilibrio entre los poderes legislativo y ejecutivo, en beneficio de este último lo que constituye un aspecto de lo que se llamó "déficit democrático" de las Comunidades Europeas. Por supuesto el Estado comunitario adquiría una permeabilidad casi absoluta respecto del Derecho creado por la nueva organización. Más tarde el impacto comunitario se percibió en los problemas que iban a surgir en los Estados compuestos en torno a la articulación entre Federación y Estados federados o, en el caso de España, Estado y Comunidades Autónomas. Incluso quedaban afectados conceptos que parecían estar al margen de la integración europea como, por ejemplo, el de la Nación, que adquiría nuevos contenidos cuando el Tratado de la Unión Europea introdujo la categoría nueva de la ciudadanía europea. Al mismo tiempo, ese mismo Estado, a través de sus representantes en el Consejo de Ministros, participaba en las decisiones comunitarias junto con los demás Estados miembros. De esta manera, el Estado clásico, que hasta entonces era capaz de actuar soberanamente en su ámbito, aceptaba límites en el ejercicio de su soberanía para adoptar decisiones en las materias que se habían encomendado a las Comunidades Europeas. Ya no podía decidir aisladamente sino que debía acompasar la defensa de sus intereses con los demás Estados comunitarios. Es decir, el Estado, no directamente, sino a través de su presencia en las Comunidades Europeas decidiría sobre esas cuestiones mancomunadamente, o sea de modo comunitario.

Lo decía muy bien el Proyecto de Constitución Europea, ya decaído, en su artículo 1.1.: "La Unión... ejercerá, de modo comunitario, las competencias que éstos (los Estados) le transfieran").

Una parte de los Estados europeos se organizaron un día en las Comunidades Europeas para salvaguardar valores comunes y hoy continúa renovando día a día esa organización a través de la legislación comunitaria ordinaria o, a veces, de modo extraordinario mediante el tratado, especialmente a partir del Acta Única Europea. Esta actualización constante le permite incorporar nuevos valores y fines a su organización, llamada hoy Unión Europea.

La nueva dimensión comunitaria ha supuesto para el Estado una transformación que se puede llamar estructural. Y antes de continuar quisiera precisar qué entiendo por "transformación estructural del Estado".

Los Estados, en tanto que artefactos para la organización social, están en constante movimiento y transformación en función de las necesidades y valores considerados por quienes los dirigen (un príncipe, una clase social, la mayoría del pueblo...). Así, desde su nacimiento en el siglo xv hasta ahora, el Estado ha sufrido con carácter general unas transformaciones de hondo calado que, además de permanecer en el tiempo, han tenido el impacto suficiente como para que la doctrina científica las haya elevado a la condición de categorías. De esta forma, se puede afirmar que el Estado, que nació como absoluto, que cristalizó en Estado de Derecho tras las revoluciones liberales y que otras revoluciones lo convirtieron en democrático, más tarde, de la mano de la democracia, el Estado abstencionista se transformó en social. 
El eje de las primeras transformaciones es la soberanía. En el Estado absoluto, la soberanía correspondía al Monarca, en el Estado liberal la soberanía corresponde a la Nación y el Estado democrático nace cuando el poder soberano corresponde al pueblo. Desde este punto de vista, el Estado social es una consecuencia en Europa del carácter democrático del Estado y el criterio del cambio, en este caso, es una nueva concepción de la interacción entre el Estado y la sociedad.

También la creación de la Unión Europea ha transformado sustancialmente la situación del Estado y la de sus propias instituciones, como se ha visto y se comprueba día a día.

Los conceptos aludidos anteriormente, de Estado de Derecho, democrático y social constituyen categorías perfectamente definidas aunque puedan sufrir ciertos cambios que exijan matizar los conceptos. En cambio, no sucede lo mismo respecto del carácter comunitario del Estado. En la medida en que estamos siendo testigos de la transformación del Estado en un sentido comunitario, desconocemos el perfil último de esta categoría, aunque sí podemos dar cuenta de algunas de las transformaciones sufridas por el Estado. Es el caso, por ejemplo, de aquella por la que adopta alguna de sus decisiones de modo mancomunado o "comunitario", con los demás Estados miembros. Es también la aplicabilidad directa del Derecho de la Unión. O el sometimiento al Tribunal de Justicia de las Comunidades Europeas cuyas sentencias son también aplicables directamente sin la posibilidad de aplicar el procedimiento de exequatur.

Anteriormente me he referido al hecho de que con su incorporación a la Unión Europea, el Estado aceptaba límites en el ejercicio de su soberanía. Y así es, ya que no es posible hablar de una cesión de soberanía a la nueva asociación de Estados, puesto que el Estado siempre tiene la posibilidad de abandonar la Unión ante una decisión que no pudiera asumir. Pero, mientras permanece como miembro, y no es fácil dejar de hacerlo por las implicaciones de todo tipo que tendría una salida de la Unión, debe ceder el ejercicio de determinadas decisiones a la nueva organización.

El eje en torno al que gira esta transformación sería el nuevo modo de trabajar mancomunado o comunitario de los Estados miembros en el seno de la Unión y la necesaria transferencia de la capacidad de adoptar decisiones del Estado a la misma Unión. En definitiva, el eje sería no la soberanía, como tal, que permanece en el Estado, sino los límites aceptados para su ejercicio.

Una conclusión que podría extraerse de lo dicho hasta ahora es, a mi juicio, que esta nueva realidad de la que somos testigos, al menos en Europa, debe de tener forzosamente consecuencias directas sobre el modo de concebir al Estado, sobre su teoría. El Estado ya no podrá ser visto como una burbuja idealmente aislada en su marco histórico nacional. Al contrario, es un Estado permeable, como se ha dicho antes, cuyos perfiles jurídicos van haciéndose más difusos y en el que sus elementos tradicionales, el pueblo y el territorio, y algunas de las decisiones que nacen de su poder soberano son compartidas con los demás Estados en el seno de la estructura superior de la Unión Europea que el mismo ha creado. 


\section{II.2. EL CONCEPTO DE ORDENAMIENTO JURÍDICO}

Quisiera plantear otra consideración metodológica, que propongo con cierto temor de cometer un pecado de obviedad, y es que creo que los constitucionalistas debemos abordar el estudio de estas nuevas realidades valiéndonos, ante todo, del método jurídico.

Como ya se ha dicho, la nueva realidad en que se encuentra el Estado es que, además de él, como creador de Derecho, existen otras organizaciones que hacen lo mismo. Es la poliarquía de que he hablado antes.

A este respecto, recientemente se viene hablando de la "gobernanza multinivel». Esta expresión pretende ser una categoría que explique la situación, relaciones y ejercicio de poderes en el seno de la nueva realidad constituida por la Unión Europea y los Estados miembros. Una aproximación con la que se pretende dar una interpretación global a la situación, las relaciones y el ejercicio de los poderes en el seno de esta nueva realidad europea. Se trata de llevar al lector o al estudioso a imaginar la situación y funcionamiento de la Unión como formado por un sistema político en el que varios niveles de poder participan en el desarrollo de las funciones que aquél ejerce. El sistema se complica todavía más cuando los Estados miembros de la Unión son federales puesto que se intercala un nuevo nivel. No voy a negar la utilidad de esta forma de ver las cosas. Ciertamente, desde un método funcionalista, los Estados y la unión Europea y, en su caso, las Comunidades Autónomas, pueden observarse como un sistema que desarrolla ciertas funciones. Se pone el acento en las funciones.

Pero esta visión, propia de la Ciencia Política, va acompañada de una apreciación confusa de la nueva realidad europea que tiende a minimizar, incluso a despreciar, el papel del Estado en el seno de la Unión (En este sentido, se puede ver el clarísimo ejemplo que nos ofrece el trabajo de Liesbert Hooghe y Gary Marks: Gobernanza estatocéntrica y gobernanza multinivel, en Francesc Morata (Ed.): Gobernanza multinivel en la Unión europea, Valencia, 2004). Incluso la propia expresión "gobernanza multinivel" deja bien claro el intento de sumergir confusamente al Estado en un conjunto de niveles desiguales. ¡El Estado que, por su carácter soberano, está en el centro de todas las organizaciones que se construyen mediante el acuerdo entre varios Estados;

Es indudable que hay un espacio político más allá del Estado. Y que este espacio requiere una especial atención por la intensidad que alcanza la vida política en él, especialmente en lo que se refiere a la Unión Europea. Pero ello no nos debe llevar al olvido de que la propia Unión Europea es una organización creada e integrada por sus propios Estados miembros.

Creo que sería un error que los constitucionalistas pensáramos que la aproximación a nuestro objeto de estudio a través de la gobernanza multinivel constituye una visión o enfoque indispensable para los trabajos de un jurista.

Tratar de traer a nuestra disciplina jurídica este enfoque propio de la Ciencia Política sería un ejercicio bastante inútil. Los constitucionalistas, y los juristas en general, tenemos ya un enfoque propio y multisecular. Un enfoque que ha 
sido perfeccionado gracias a las aportaciones de las grandes escuelas nacionales del Derecho Público europeas, como la alemana, la francesa, la italiana... A propósito de esta última, quisiera subrayar la aportación de Santi ROMANO sobre el concepto de ordenamiento jurídico y de la dinámica de las relaciones entre los diferentes ordenamientos jurídicos en presencia. Este concepto nos permite ver las realidades que nos interesan con una gran precisión a partir de la distribución de competencias entre ellos, es decir, teniendo como eje central del análisis el principio de competencia.

Ambos enfoques, el de la gobernanza multinivel y el jurídico, pueden llevarnos a visiones de los problemas que no tienen por qué ser ni contradictorias ni divergentes. Al contrario, si se manejan bien, nos tendrían que llevar ambas a una visión similar. Y digo similar y no igual porque cada enfoque nos muestra distintas realidades de un mismo objeto. El enfoque jurídico nos va a dar cuenta de realidades jurídicas. El enfoque político nos dará cuenta de realidades políticas. El primero es indispensable para el Derecho puesto que es el único que nos permite afinar los datos contemplados con el fin de dar soluciones tan necesariamente matizadas y sutiles como las que exige una sentencia judicial. La visión política puede dar soluciones de trazo más grueso, pero que serán útiles para los fines propios de la Ciencia Política y también, como medio de conocimiento (¿porqué no?), para los juristas.

Como se sabe, el concepto de ordenamiento jurídico es equivalente al de organización social. De modo que cada uno de estos ordenamientos jurídicos es una organización social que se dan a sí mismos los distintos pueblos (de los Estados o de las entidades regionales afectadas) en torno a sus centros de poder, o sea, los órganos que crean el Derecho en cada una de esas organizaciones sociales. De este modo, la pluralidad de organizaciones sociales que constituyen los Estados miembros, la Unión Europea y, eventualmente, en el caso de los Estados compuestos, las regiones, Comunidades Autónomas o Länder, es, en realidad, una pluralidad de ordenamientos jurídicos que se relacionan entre ellos de acuerdo con las competencias que a cada uno de ellos corresponden según la distribución que se haya hecho en las Constituciones, Estatutos de Autonomía y en los Tratados reguladores de la Unión.

De esta aproximación jurídica hay que destacar fundamentalmente la claridad y precisión de los resultados que se obtienen a partir de ella frente a las afirmaciones carentes de esas virtudes que resultan de otras aproximaciones metodológicas.

En este sentido, pueden verse las imprecisas afirmaciones que se hacen por los autores Liesbert Hooghe y Gary Marks, que antes he citado, cuando, por ejemplo, plantean dos concepciones alternativas básicas de la integración europea, "la gobernanza estatocéntrica y gobernanza multinivel" de manera que según su percepción, de acuerdo con el modelo estatocéntrico, la Unión europea no es más que el resultado de una continua negociación entre los ejecutivos de los Estados miembros y, en cambio, desde la perspectiva de la gobernanza multinivel, "la integración europea es una estructura política que crea un proceso en el que la autoridad y la influencia a la hora de elaborar las decisio- 
nes están compartidas entre múltiples niveles de gobierno subnacional, nacional y supranacional" (p. 52 del trabajo citado anteriormente).

Por el contrario, cuando se analiza la Unión Europea desde el método jurídico, el resultado final, que probablemente lo vamos a tener en forma de sentencia, no tiene porqué hacer afirmaciones acerca de si es mucho o poco lo que los Estados influyen en las decisiones de la Unión Europea, sino que obtenemos con total precisión si, en un caso concreto, la Unión Europea podía adoptar la decisión objeto del litigio o no, o, si por el contrario, el Estado realizó correctamente una actuación concreta o no.

\section{CONCLUSIONES}

A mi juicio, esta nueva realidad de la que somos testigos, al menos en Europa, debe de tener consecuencias directas sobre la propia teoría del Estado, sobre el modo de concebirlo y explicarlo jurídicamente. El Estado, en el marco de la organización policéntrica de nuestras sociedades ya no puede ser estudiado como una burbuja idealmente aislada en su marco histórico nacional. Al contrario, es un Estado permeable, cuyos perfiles jurídicos van haciéndose más difusos y en el que sus elementos tradicionales, el pueblo y el territorio, y algunas de las decisiones que nacen de su poder soberano, son compartidas con los demás Estados en el seno de la estructura superior de la Unión Europea que el mismo ha creado. En definitiva, el Estado ha adquirido una nueva dimensión, especialmente intensa en el ámbito de la Unión Europea.

Por otra parte, aún a riesgo de insistir en lo obvio, creo que el método jurídico, especialmente a partir del análisis llevado a cabo sobre la base del concepto de ordenamiento jurídico y del principio de competencia, constituye la manera más adecuada de trabajar para el constitucionalista en una cuestión en la que, como ésta, se dan cita diversos ordenamientos jurídicos.

Finalmente, creo que unas posibles directrices futuras en relación con los nuevos estudios de Derecho o las medidas que se adopten a raíz del proceso de adaptación al espacio europeo de educación superior, deberían tener en cuenta las conclusiones de las transformaciones del Estado de que se habla en estas páginas. En cuanto al Derecho Constitucional, en concreto, deberían hacer referencia al impacto que el ordenamiento comunitario tiene sobre los ordenamientos de los Estados miembros. Lo anterior probablemente conduzca a la conclusión de que los contenidos de la asignatura Instituciones de Derecho Comunitario deberían ser trasladados a sus lugares "naturales", el Derecho Administrativo, Constitucional e Internacional Público como ya ocurre hoy con el Derecho material comunitario referido a las distintas materias mercantiles, civiles o penales que se investigan y estudian desde el Derecho Mercantil, el Derecho Civil o el Derecho Penal sin necesidad de que exista un Derecho Comunitario Mercantil, Civil o Penal 
ABSTRACT. The author meditates about the necessity of approaching in a new way the reality of a transformed State as member of the European Union and in the new surroundings of a globalized world. He analyzes the possible future guidelines equally in connection with the new studies of Law. 